\title{
An Invitation to Categorification
}

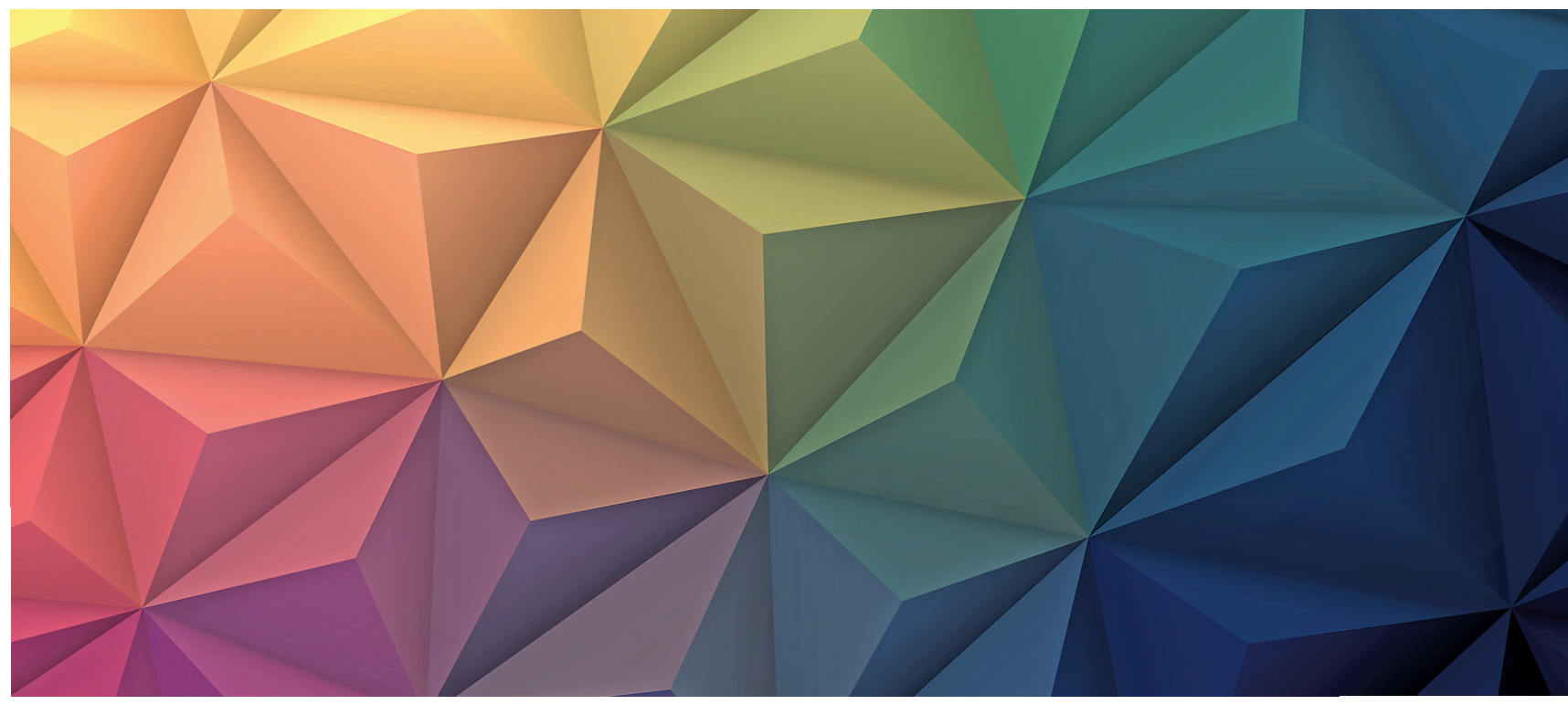

\section{Aaron D. Lauda and Joshua Sussan}

\section{What is Categorification?}

Categorification is the process of promoting an algebraic object to one with more structure. All of the structure of the original object is retained and can be accessed via a decategorification procedure that forgets this new higherlevel structure. Originally this term indicated the replacement of set-based constructions with categorical notions, where equalities are replaced by explicit isomorphisms. However, over the years, the term categorification has been used in a variety of different contexts, making its precise definition somewhat nebulous. In most cases, one specifies a rigorous "decategorification" procedure that forgets some type of structure. Categorification is then the inverse process of trying to find the more sophisticated structure that would decategorify to some specified object of interest. Within this broad framework are some guiding principles

Aaron D. Lauda is a professor of mathematics at the University of Southern California. His email address is 1 auda@usc.edu.

Joshua Sussan is a professor of mathematics at CUNY Medgar Evers and the CUNY Graduate Center. His email addresses are jsussan@mec.cuny.edu and jsussan@gc.cuny.edu.

For permission to reprint this article, please contact: reprint-permission@ams.org.

DOI: https://doi.org/10.1090/noti2399 one usually has in mind when pursuing categorification:

- enhancement of algebraic structure,

- precise but context-dependent notion of decategorification,

- richer structure not seen in the original objectleading to a deeper understanding of the original object.

In this article we will give a variety of examples to help illustrate these points. We begin with some fairly trivial examples of categorification, building up to more sophisticated ones.

Example 1 (Categorification of natural numbers). The category of finite-dimensional vector spaces over a field $\mathbb{k}$ can be viewed as a categorification of the natural numbers $\mathbb{Z}_{\geq 0}$. In this case, decategorification is the dimension map that sends a finite-dimensional vector space to its dimension. A natural number $n$ is categorified by a choice of an $n$ dimensional vector space.

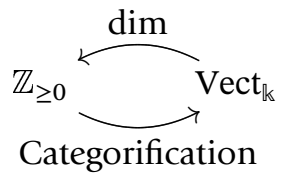

$n \rightsquigarrow n$-dimensional vector space 
The operations of addition and multiplication lift as follows:

- $\operatorname{dim}(V \oplus W)=\operatorname{dim} V+\operatorname{dim} W$,

- $\operatorname{dim}(V \otimes W)=\operatorname{dim} V \cdot \operatorname{dim} W$.

Example 2 (Categorification of Laurent polynomials). The category of finite-dimensional $\mathbb{Z}$-graded vector spaces can be used to categorify Laurent polynomials $f \in$ $\mathbb{Z}_{\geq 0}\left[q, q^{-1}\right]$. Recall that a $\mathbb{Z}$-graded vector space decomposes into a direct sum, so that its graded dimension is given by:

$$
V=\bigoplus_{n \in \mathbb{Z}} V_{n}, \quad \operatorname{gdim} V:=\sum_{n \in \mathbb{Z}} q^{n} \operatorname{dim} V_{n}
$$

In this case, decategorification is taking the graded dimension. Then, $f \in \mathbb{Z}_{\geq 0}\left[q, q^{-1}\right]$ is categorified by a graded vector space $V$, where the coefficient of $q^{n}$ is the dimension of $V_{n}$, and $f$ is realized as the graded dimension $\operatorname{gdim} V$.

$$
\begin{aligned}
& \mathbb{Z}_{\geq 0}\left[q, q^{-1}\right] \quad \text { GrVect }_{\mathbb{k}} \\
& \text { Categorification } \\
& f \leadsto \text { graded vector space } V
\end{aligned}
$$

Note that in these two examples, we cannot realize a negative coefficient of $q^{n}$ using graded vector spaces, since the dimension of a vector space is never negative. If we want to include negative coefficients, we need a more sophisticated categorification.

Example 3. Suppose we are given a complex of graded vector spaces

$$
V^{\cdot}=\cdots \longrightarrow V^{i} \stackrel{d}{\longrightarrow} V^{i+1} \stackrel{d}{\longrightarrow} V^{i+2} \longrightarrow \cdots .
$$

Then decategorification is defined to be the graded Euler characteristic

$$
\chi\left(V^{\cdot}\right):=\sum_{i \in \mathbb{Z}}(-1)^{i} \operatorname{gdim} V^{i} .
$$

Note that a Laurent polynomial $f \in \mathbb{Z}\left[q, q^{-1}\right]$ can be lifted to a chain complex in many ways. Indeed, we can always find a lifting with trivial differentials. However, in practice, the naturally occurring examples usually have nontrivial differentials, indicating a richer structure.

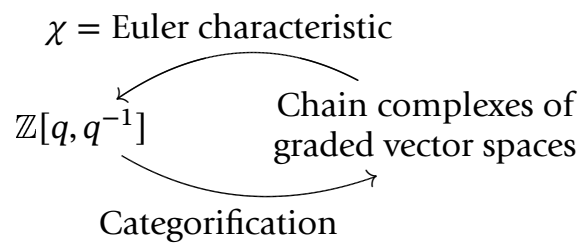

The examples given above illustrate some of the features of categorification, but they fail to illustrate how categorification can bring in new information. The idea of categorification is actually very classical, going back to the origins of algebraic topology. For example, singular homology groups provide a categorification of the Euler characteristic. There is more topological information contained in homology groups, and these invariants are functorial with respect to continuous maps between spaces.

Example 4. For an $n$-dimensional CW complex $X$ given by gluing $k_{i} i$-cells for $i \leq n$, the Euler characteristic is defined as

$$
\chi(X)=k_{0}-k_{1}+k_{2}-k_{3}+\cdots=\sum_{i=0}^{\infty}(-1)^{i} k_{i} .
$$

For example, consider the sphere decomposed as a CW complex:

$$
x(\leadsto)=2
$$

For any cell decomposition of the 2-sphere (such as the one depicted above), we have

$$
\#(\text { vertices })-\#(\text { edges })+\#(\text { faces })=2 \text {. }
$$

The Euler characteristic is categorified by the homology groups $H_{i}(X)$.

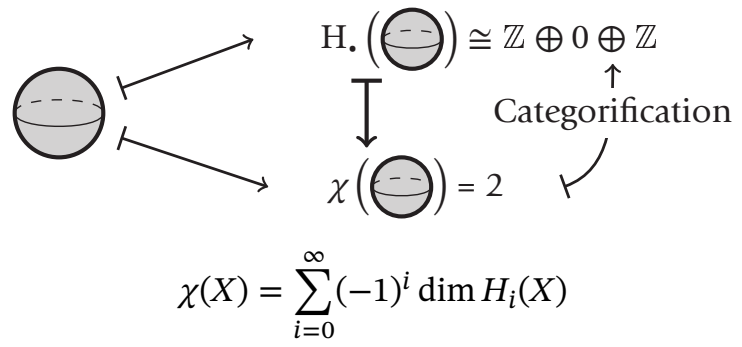

What are the advantages of homology over the Euler characteristic?

- Homology groups are better invariants of spaces. (More spaces can be distinguished by homology groups than by Euler characteristic.)

- Homology is functorial: given $f: X \rightarrow Y$ we get maps between homology $f_{i}: H_{i}(X) \rightarrow H_{i}(Y)$. This functoriality has many important applications, such as a proof of Brouwer's fixed-point theorem.

Looking at the examples above, the reader may be wondering where the categories appear in "categorification." The next example helps illustrate how all of these examples above fit into a unified picture.

Example 5 (Grothendieck group of a category). Let $C$ be an additive category. This just means that the category has direct sums and an object $\mathbf{0}$ with the expected properties. The Grothendieck group is a way to decategorify, reducing a category to a set. In this case, the set has the additional structure of an abelian group. The (split) Grothendieck group of $\mathcal{C}$ is defined to be the free abelian group on the isomorphism classes $[X]$ of objects $X$, modulo the relations $[X \oplus Y]=[X]+[Y]$. If the category has a monoidal 
structure, then the Grothendieck group inherits a multiplicative structure and we thus obtain a Grothendieck ring. For example, since every finite-dimensional $\mathbb{k}$-vector space is isomorphic to some $\mathbb{k}^{n}$, the Grothendieck group $K_{0}\left(\mathrm{Vect}_{\mathrm{k}}\right)$ is the free abelian group on the generator [k], and for a general vector space we have $[V]=\operatorname{dim} V[\mathbb{k}]$. In particular, the decategorification of a vector space by taking its dimension is just a special case of $K_{0}$.

Similarly, if the category $\mathcal{C}$ is graded, so that it is equipped with a grading shift autoequivalence $\langle 1\rangle: \mathcal{C} \rightarrow \mathcal{C}$ (such as the category of graded vector spaces), then the Grothendieck group can be equipped with the structure of a $\mathbb{Z}\left[q, q^{-1}\right]$-module by declaring that $[X\langle 1\rangle]=q[X]$. Applying $K_{0}$ to the graded additive category of graded vector spaces, we now see that $K_{0}\left(\right.$ GrVect $\left._{\mathrm{k}}\right)$ is generated as a free $\mathbb{Z}\left[q, q^{-1}\right]$-module by $[\mathbb{k}]$ and that for a general graded vector space $[V]=\operatorname{gdim} V \cdot[\mathbb{k}]$. The example of taking the graded Euler characteristic of a complex of graded vector spaces from Example 3 can also be understood as the Grothendieck group applied to an appropriate category of complexes of graded vector spaces.

Categorifying other rings, such as cyclotomic rings or $\mathbb{Z}\left[\frac{1}{N}\right]$, is a very difficult problem. We will mention later on the importance of categorifying cyclotomic rings and progress in this direction. Khovanov and Tian constructed monoidal categories whose Grothendieck rings are isomorphic to $\mathbb{Z}\left[\frac{1}{2}\right]$, and offer some perspectives on the more general question.

\section{Origins of the Categorification Philosophy}

In this section we see how state-sum TQFTs inspired Crane and Frenkel's categorification program [CF94].

2.1. TQFTs. An $n$-dimensional topological quantum field theory (TQFT) is a (symmetric monoidal) functor $F$ from the category $n \mathrm{Cob}$ of $n$-dimensional cobordisms to the category of vector spaces. This amounts to a rule associating a vector space to each (closed, oriented) $(n-1)$ manifold and a linear map to each $n$-manifold with boundary (a cobordism between the boundary $(n-1)$ manifolds). Functoriality ensures that composing cobordisms by gluing along common boundaries gets mapped to the composition of the associated linear maps. The monoidal property of the functor means that the rule must send disjoint unions of $(n-1)$-manifolds $X \coprod Y$ to tensor products $F(X) \otimes F(Y)$ of vector spaces, and the disjoint union of cobordisms to the tensor product of the associated linear maps; it also means that the empty manifold must get mapped to the trivial vector space.

For example, a 2-dimensional TQFT assigns to each closed, oriented, 1-manifold (i.e., a disjoint union of circles) a vector space. Because the TQFT must preserve disjoint unions, its value on 1-manifolds is completely determined by the vector space $V$ assigned to a circle. Then a disjoint union of $n$ circles must get mapped to $V^{\otimes n}$. To any oriented 2-dimensional cobordism between 1-manifolds, the TQFT assigns a linear map.

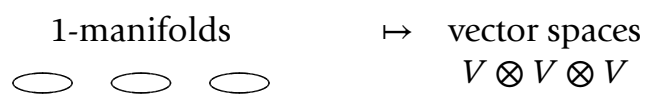

2-manifolds with boundary $\mapsto \quad$ linear maps
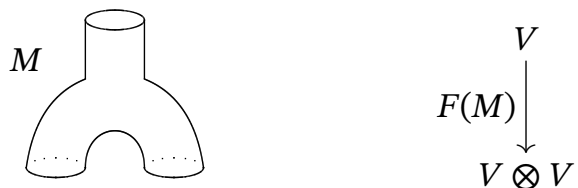

Using Morse theory, any 2-dimensional cobordism can be decomposed into elementary cobordisms (pair of pants, birth and death of a circle). This makes the algebraic description of 2D TQFTs much easier because we can give a generators and relations description of $2 \mathrm{Cob}$ by classifying critical points of the Morse function and then writing down all handle slides and cancellations. For example, we could decompose the 2-dimensional cobordism:
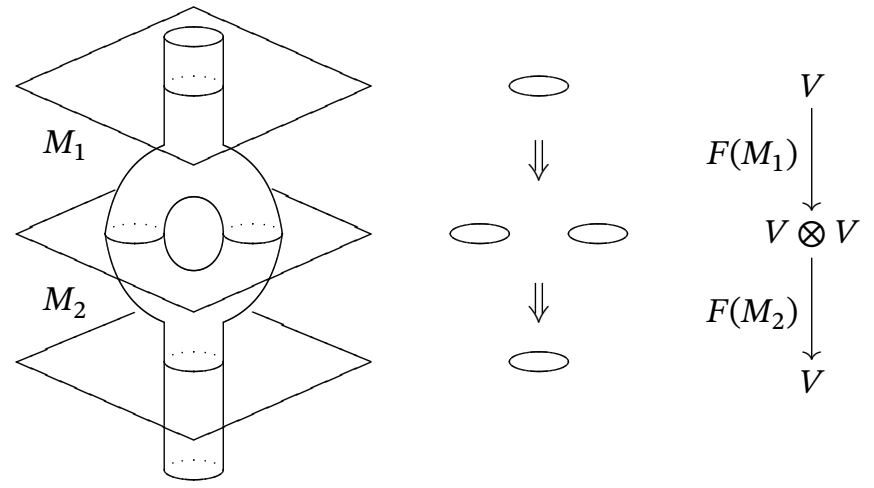

Beyond dimension 2, constructing nontrivial examples ofTQFTs can be very challenging. Dimension 4 has proven particularly challenging. Crane and Frenkel's idea was to observe how the algebraic structures needed to construct invariants change as one increases the dimension. Observing that passing from 2-dimensional TQFTs to 3dimensional ones requires moving from algebras (or vector spaces with multiplicative structure) to monoidal categories (or categories with multiplicative structure), Crane and Frenkel realized that the passage from dimension 3 to dimension 4 could similarly be achieved by increasing the categorical complexity of the algebraic inputs.

The focus on 4-dimensions in the Crane and Frenkel program stems from the goal of constucting new algebraic invariants sensitive to exotic smooth structures in 4-dimensions. The hope was that categorification could produce news tools, beyond gauge theory, for proving (or disproving) the smooth 4-dimensional Poincaré conjecture, arguably the most prominent open problem in topology. In Section 4.2 we give some indications that 

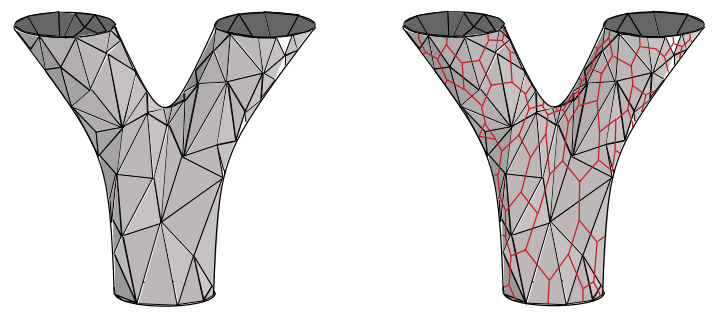

Figure 1. A triangulation of a 2-dimensional cobordism with 1-manifold boundary circles and its Poincaré dual graph.

categorification can produce new invariants that behave differently than their gauge-theoretic analogues.

2.2. State-sum 2D TQFTs. This increase in categorical complexity with increasing dimension could be understood in the context of certain local constructions of TQFTs from triangulations, or what are sometimes called statesum TQFTs. To construct local 2D TQFTs, we decompose the surface into triangles, and assign algebraic data to the triangulation. The algebraic data should be invariant under local change of triangulation.

In order to verify that the invariant is independent of triangulation, one simply needs to check invariance under the following 2D Pachner moves that relate any two triangulations of the same 2-manifold:

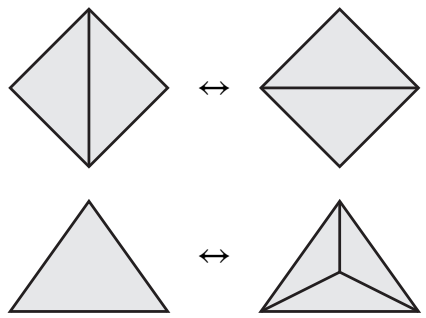

To construct an algebraic invariant from a triangulation, consider the Poincaré dual of the triangulation.

- Label each edge of the dual graph by a basis vector of a vector space $V$.

- Regard each triangle as a multiplication of basis vectors and associate to each triangle the structure constant of this multiplication. ${ }^{1}$

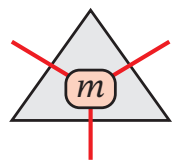

- Sum over all ways of assigning basis vectors to edges, noting that the contribution of a given state of edge assignments may be zero if any one of the multiplicative structure constants associated to a triangle is zero.

\footnotetext{
${ }^{1}$ The reader may be wondering how to decide which edges are the input and which is the output. It turns out that the choice does not matter, as we will be required to fix an isomorphism $V \cong V^{*}$ which can be used to turn inputs into outputs and vice versa; see [BL11] and the references therein for more details.
}

Invariance under triangulation requires

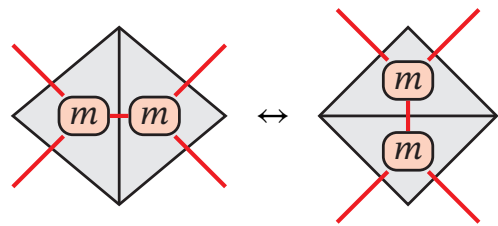

or

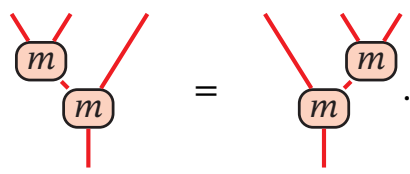

This is the axiom of associativity. It requires that the vector space labelling edges has an associative algebra structure. The other Pachner move implies that the algebra must be semisimple. $^{2}$

State sums in 3-dimensions work similarly to the 2dimensional case. Now, we need to assign data to each tetrahedron of a triangulated 3-manifold. We again take Poincaré duals, but in 3-dimensions these dual graphs produce 2-complexes (or 2-dimensional graphs).

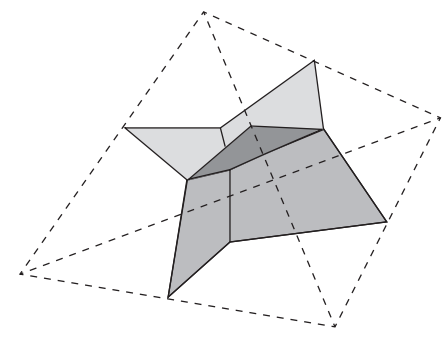

Going from the back to the front of this picture we can think of the tetrahedron as supplying a map from the graph on one side to the graph on the other:
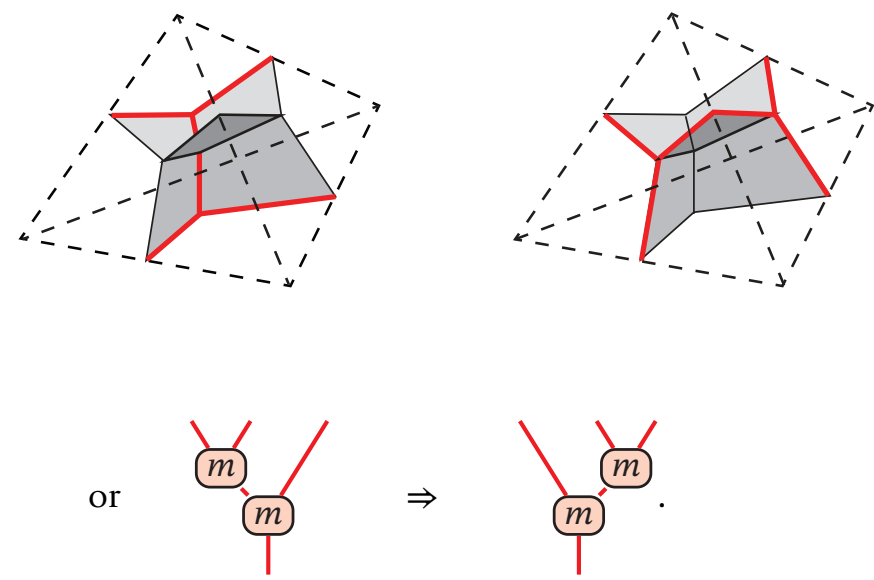

Associativity is no longer an equation; it is now an isomorphism.

\footnotetext{
${ }^{2}$ Technically, the algebra must be strongly separable. These two notions are equivalent over fields of characteristic 0 .
} 
Invariance under triangulation in 3-dimensions is ensured by 3D Pachner moves, one of which is the 2-3 Pachner move:

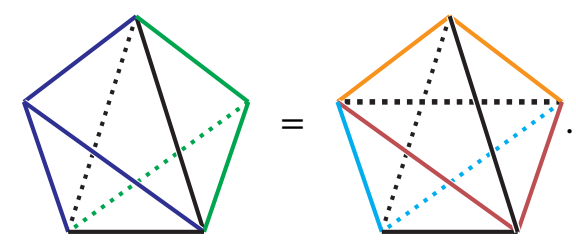

It is called the 2-3 move because it changes from a triangulation involving two tetrahedra into one involving three.

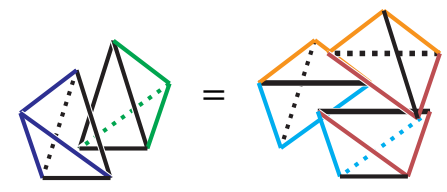

Viewing each tetrahedron as a process of changing a 2dimensional triangulation, we can view each tetrahedron appearing in the 2-3 move as a "movie" depicting a sequence of 2-dimensional Pachner moves changing the triangulation as we pass through each tetrahedron. The 23 move can then be viewed as an equality between two sequences of 2-dimensional triangulation changes as in Figure 2. But this equation is exactly the associator condition used in the definition of monoidal categories. The other 3D-Pachner move imposes additional constraints on the category that can be viewed as categorifications of the semisimple criteria of an algebra. For further details the reader is referred to the exposition in [BL11].

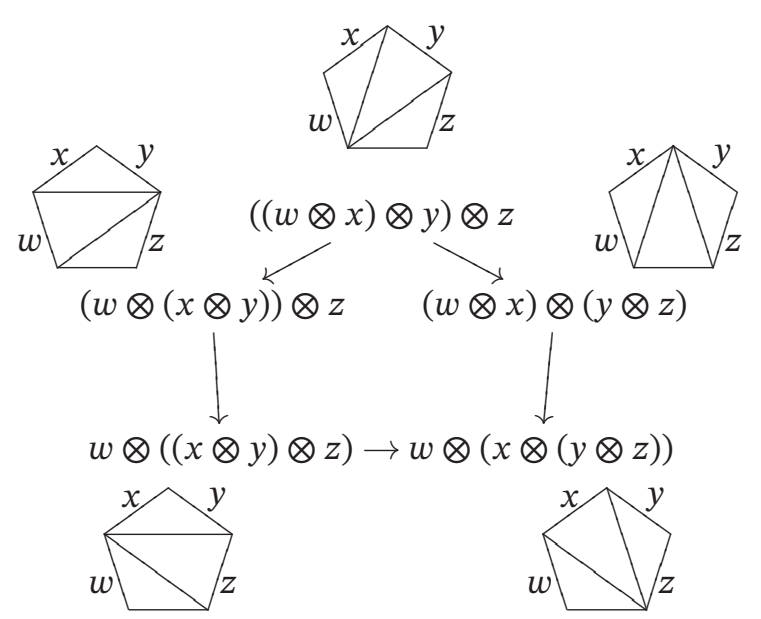

Figure 2. The associator as change of triangulation.

2.3. Observations. State-sum TQFTs suggest that one needs to increase the categorical complexity, or categorify, as one increases the dimension. In 3-dimensions, to assign data to a triangulation we need a categorical analog of an algebra, i.e., a monoidal (or tensor) category.

$$
\begin{array}{lll}
\frac{\text { 2-dim }}{\text { Algebra }+} & \underline{\text { 3-dim }} & \underline{\text { 4-dim }} \\
\text { extra structure } & \begin{array}{l}
\text { multiplication } \\
\text { + extra structure }
\end{array} & \begin{array}{l}
\text { 2-Category with } \\
\text { multiplication }
\end{array} \\
& \text { extra structure }
\end{array}
$$

Crane and Frenkel's idea is to bootstrap up the dimensional ladder of $n$-dimensional TQFTs by categorifying the relevant algebraic structures. Baez and Dolan then extended these ideas to formulate the cobordism hypothesis relating $n$-dimensional TQFTs to certain $n$-categories with extra structure. These ideas where later verified in the work of Lurie.

This then raises the question of where to find good examples of the relevant structures in each dimension. In dimension 3, Reshetikhin, Turaev, and Viro showed that the theory of quantum groups provides such examples connecting to Witten's work in Chern-Simons gauge theory. Part of what made Crane and Frenkel's proposal so compelling is that they not only suggested increasing the categorical complexity as one raises the dimension of the TQFT, they also gave convincing evidence that it should be possible to categorify the theory of quantum groups using various Lie theoretic and geometric methods, giving rise to new 4-dimensional TQFTs. These ideas birthed the field of categorification.

\section{Quantum Groups}

Quantum groups provide an algebraic input that can be used to define a 3-dimensional TQFT. Relatedly, representations of quantum groups give rise to invariants of links. We begin with a combinatorial description of one such link invariant, before connecting it to quantum group theory.

3.1. The Jones polynomial. The Jones polynomial is an invariant of oriented links. This invariant can be computed from a simple recursive algorithm, called the Kauffman bracket, defined on link diagrams as follows:

1. $\langle\emptyset\rangle=1$

2. $\langle L \coprod \bigcirc\rangle=\left(q+q^{-1}\right)\langle L\rangle$

3. \langle\rangle\langle\rangle$=\langle||\rangle-q\langle\stackrel{\sim}{\sim}\rangle$

An example of how to implement this recursive algorithm on a knot is illustrated in Figure 3.

The Jones polynomial is a rescaling of the Kauffman bracket $J(L)=c\langle L\rangle$, where $c$ is determined from an orientation of the knot. This invariant is a special case of a vastly more general set of invariants associated to quantum groups.

3.2. What are quantum groups? A quantum group refers to a Hopf algebra with some extra structure which makes its category of representations useful for studying knots. Important examples of quantum groups are Hopf algebras 


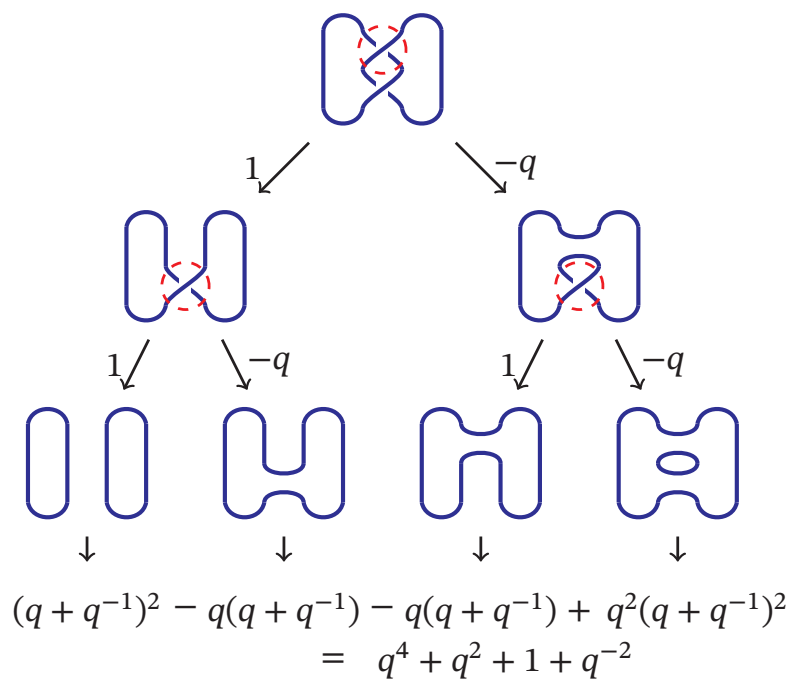

Figure 3. Implementing the Kauffman bracket algorithm on a Hopf link.

$U_{q}(\mathfrak{g})$ associated to simple Lie algebras $\mathfrak{g}$ :

- Their representations behave much like representations of the corresponding Lie algebra.

- They have very nice categories of representations (braided monoidal + duals).

- They can be described explicitly using generators and relations.

One of the simplest examples of a quantum group is the quantum group associated to $\mathfrak{g}=\mathfrak{S l}_{2}$. The quantum group $U_{q}\left(\mathfrak{S I}_{2}\right)$ is the associative algebra over $\mathbb{C}(q)$ with generators $E, F, K, K^{-1}$ and relations

- $K K^{-1}=1=K^{-1} K$,

- $K E=q^{2} E K, \quad K F=q^{-2} F K$,

- $E F-F E=\frac{K-K^{-1}}{q-q^{-1}}$.

3.3. Reshetikhin-Turaev invariants. We now outline how quantum groups give rise to link (and more generally tangle) invariants. Start with a tangle diagram $T$ and a Lie algebra $\mathfrak{g}$ :

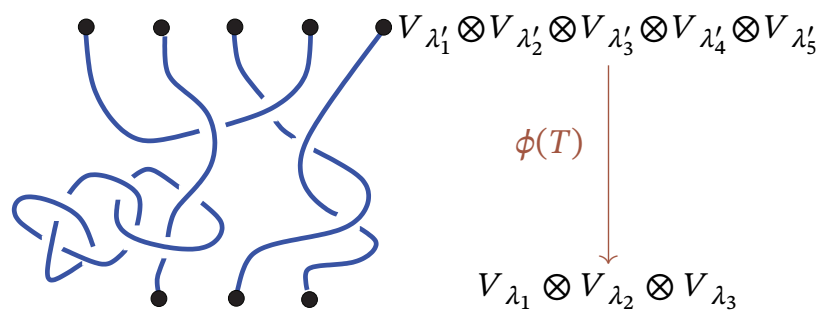

- Label the strands of the tangle by irreducible representations of $U_{q}(\mathfrak{g})$. This induces a labelling of the endpoints.

- The Reshetikhin-Turaev invariant gives a morphism of $U_{q}(\mathfrak{g})$-representations associated to the tangle diagram. One decomposes the tangle diagram into elementary pieces so that each piece is either a single crossing, cup, or cap. To the crossings one associates the braiding in the category, while cups and caps correspond to duality structures in the category.

- Reshetikhin and Turaev show that the morphism does not depend on the planar projection of the tangle.

For a knot, the resulting morphism is a map from the ground field to itself. Such maps correspond to elements of the ground field.
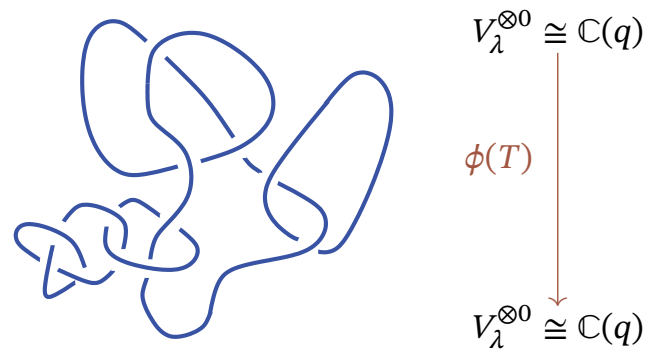

It turns out that these invariants always land in $\mathbb{Z}\left[q, q^{-1}\right]$, so we get a Laurent polynomial. For $\mathfrak{g}=\mathfrak{s l}_{2}$, we obtain the Jones polynomial, and more generally colored Jones polynomials. If $\mathfrak{g}=\mathfrak{s l}_{n}$, we obtain specializations of the colored HOMFLYPT polynomial.

3.4. Connections to TQFTs. There are two related ways of using representations of quantum groups to define 3dimensional TQFTs. Both constructions require specializing the quantum parameter $q$ to be a root of unity to ensure certain finiteness properties, so that the sums involved converge.

Any closed 3-manifold may be obtained by performing surgery on a link in $S^{3}$. By assigning sums of (colored) Jones polynomials of the link, Reshetikhin and Turaev obtained an invariant of the 3-manifold. This could then be extended to a 3-dimensional TQFT.

Turaev and Viro defined an invariant of 3-manifolds by assigning quantum $6 j$ symbols to the tetrahedra of a triangulation, and summing over all such assignments. This state-sum invariant has also been extended to a TQFT.

\section{Examples of Categorification}

4.1. Categorification of representations. Given the important role that quantum groups and their representations play in constructing topological invariants, following Crane and Frenkel, it was expected that categorifying these algebraic structures would lead to invariants of topological objects in higher dimensions.

One of the first examples of a categorification was a categorification of the $n$th tensor power of the natural two-dimensional $\mathfrak{S l}_{2}$-representation $V_{1}$ due to Bernstein, Frenkel, and Khovanov [BFK99]. This was generalized to 
arbitrary tensor products of irreducible representations of $\mathfrak{S l}_{2}$, and extended to the quantum setting by Frenkel, Khovanov, and Stroppel [FKS06]. These tensor products were used in Section 3.3 in the Reshetikhin-Turaev approach to the Jones polynomial and its extensions to colored Jones polynomials.

We will summarize the Lie theoretic categorification described in [BFK99] to give a high-level overview of the categorification procedure before expanding upon a concrete example that can be easily understood without Lie theoretic notions. This construction uses infinite-dimensional representations of the Lie algebra $\mathfrak{g l}_{n}$. Let $\mathcal{O}\left(\mathfrak{g l}_{n}\right)$ denote the full subcategory of $U\left(\mathfrak{g l}_{n}\right)$-modules which are

- finitely generated,

- locally finite under the action of $U(\mathfrak{b})$, where $\mathfrak{b}$ is the Lie subalgebra of upper-triangular matrices,

- diagonalizable under the algebra $\mathfrak{h}$ of diagonal matrices.

The category $\mathcal{O}\left(\mathfrak{g l}_{n}\right)$ decomposes into a direct sum of subcategories according to the action of the center $z$ of $U\left(\mathfrak{g l}_{n}\right)$. For $i=0, \ldots, n$, let $\mathcal{O}_{i}\left(\mathfrak{g l}_{n}\right)$ denote the subcategory for which $z$ acts via a generalized central character corresponding to an integral dominant weight $\lambda_{i}$ which has stabilizer $S_{i} \times S_{n-i}$ under the dot action of the Weyl group $\left(S_{n}\right.$ in this case).

There are functors

$$
\mathrm{E}: \mathcal{O}_{i}\left(\mathfrak{g l}_{n}\right) \rightarrow \mathcal{O}_{i+1}\left(\mathfrak{g l}_{n}\right)
$$

given by tensoring with the natural representation of $\mathfrak{g l}_{n}$ along with projection onto the subcategory.

Similarly, there are functors

$$
\mathrm{F}: \mathcal{O}_{i}\left(\mathfrak{g l}_{n}\right) \rightarrow \mathcal{O}_{i-1}\left(\mathfrak{g l}_{n}\right)
$$

given by tensoring with the dual of the natural representation of $\mathfrak{g l}_{n}$ along with projection onto the subcategory.

It was proved in [BFK99] that as a vector space, $\bigoplus_{i=0}^{n} K_{0}\left(\mathcal{O}_{i}\left(\mathfrak{g l}_{n}\right)\right) \cong V_{1}^{\otimes n}$, and there are isomorphisms of functors on the category $\mathcal{O}_{i}\left(\mathfrak{g l}_{n}\right)$ :

$$
\begin{array}{ll}
\mathrm{EF} \cong \mathrm{FE} \oplus 1^{\oplus 2 i-n}, \quad \text { if } 2 i-n \geq 0, \\
\mathrm{EF} \oplus 1^{\oplus n-2 i} \cong \mathrm{FE}, \quad \text { if } 2 i-n \leq 0 .
\end{array}
$$

This endows $\bigoplus_{i=0}^{n} K_{0}\left(\mathcal{O}_{i}\left(\mathfrak{g l}_{n}\right)\right)$ with the structure of an $\mathfrak{s l}_{2}$-representation isomorphic to $V_{1}^{\otimes n}$. Note that we are implicitly complexifying the Grothendieck groups by tensoring with $\mathbb{C}$ to obtain complex vector spaces (or vector spaces over $\mathbb{C}(q)$ ) to match the representation theory of the quantum group from earlier.

We will now explore the case of $n=2$, where the Lie theoretic ideas above can be described in a more downto-earth algebraic fashion. Let $C=\mathbb{C}[x] /\left(x^{2}\right)$. Let $A$ be the quotient of the path algebra of (4.1) by the two-sided ideal generated by $(1|2| 1)$.

(1)

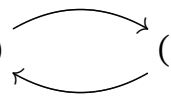

Then we consider categories $\mathcal{V}_{-2}:=\mathbb{C}-\bmod , \mathcal{V}_{0}:=$ $A-\bmod , \mathcal{V}_{2}:=\mathbb{C}-$ mod. It is easy to check that the center of the algebra $A$ is isomorphic to $C$ and can be identified with the subalgebra (2) $A(2)$. Now we construct some functors between these categories.

Let $B_{1}=(2) A$ and $B_{2}=A(2)$ regarded as $(\mathbb{C}, A)$ and $(A, \mathbb{C})$-bimodules, respectively. Then there are exact functors

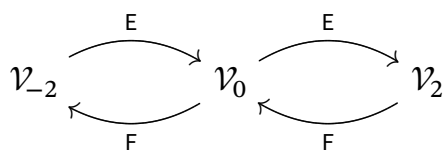

$$
\mathrm{E}: \mathbb{C}-\bmod \rightarrow A-\bmod , \quad \mathrm{E}: A-\bmod \rightarrow \mathbb{C}-\bmod
$$

given by

$$
B_{2} \otimes_{\mathbb{C}} \cdot, \quad B_{1} \otimes_{A} \cdot
$$

Similarly, there are exact functors

$$
\mathrm{F}: \mathbb{C}-\bmod \rightarrow A-\bmod , \quad \mathrm{F}: A-\bmod \rightarrow \mathbb{C}-\bmod
$$

given by

$$
B_{2} \otimes_{\mathbb{C}} \cdot, \quad B_{1} \otimes_{A} \cdot
$$

We also define $\mathrm{E}$ to be zero on $\mathcal{V}_{2}$ and $\mathrm{F}$ to be zero on $\mathcal{V}_{-2}$.

Since the functors $\mathrm{E}$ and $\mathrm{F}$ are exact, they induce an action on the Grothendieck groups:

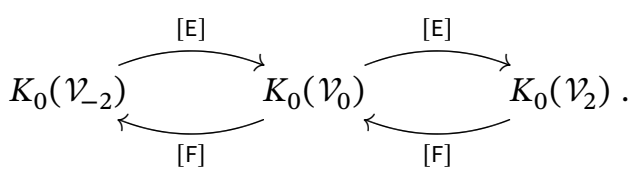

The Grothendieck groups $K_{0}\left(\mathcal{V}_{i}\right)$ of the categories above are easy to calculate. Bases of these vector spaces are given by the classes of indecomposable projective modules. Since $A \cong A(1) \oplus A(2)$ is a decomposition of $A$ as a left module over itself into indecomposable projective modules, one has $K_{0}(A-$ mod $) \cong \mathbb{C}^{2}$, while clearly $K_{0}(\mathbb{C}-$ mod $) \cong \mathbb{C}$. We may then compute the action of $[\mathrm{E}]$ and $[\mathrm{F}]$ on these basis elements. For example, on $K_{0}\left(\mathcal{V}_{-2}\right)=K_{0}(\mathbb{C}-$ mod $)$ we compute

$$
\begin{gathered}
{[\mathrm{F}] \circ[\mathrm{E}]([\mathbb{C}])=[\mathrm{F}]([A(2)])=[\mathbb{C}]+[\mathbb{C}],} \\
{[\mathrm{E}] \circ[\mathrm{F}]([\mathbb{C}])=0 .}
\end{gathered}
$$

So on $K_{0}\left(\mathcal{V}_{-2}\right)$ we see that

$$
[\mathrm{E}][\mathrm{F}]-[\mathrm{F}][\mathrm{E}]=-2 \mathrm{Id} \text {. }
$$

Similarly, on $K_{0}\left(\mathcal{V}_{2}\right)=K_{0}(\mathbb{C}-$ mod $)$ we have

$$
[\mathrm{E}][\mathrm{F}]-[\mathrm{F}][\mathrm{E}]=2 \mathrm{Id} \text {. }
$$

We could also compute on $K_{0}\left(\mathcal{V}_{0}\right)$ :

$$
\begin{gathered}
{[\mathrm{E}] \circ[\mathrm{F}]([A(2)])=[\mathrm{E}]([\mathbb{C}]+[\mathbb{C}])=2[A(2)],} \\
{[\mathrm{E}] \circ[\mathrm{F}]([A(1)])=[\mathrm{E}]([\mathbb{C}])=[A(2)] .}
\end{gathered}
$$


Similar calculations for $[\mathrm{F}] \circ[\mathrm{E}]$ on $K_{0}\left(\mathcal{V}_{0}\right)=K_{0}(A-$ mod $)$ yield the equality of operators

$$
[\mathrm{E}] \circ[\mathrm{F}]=[\mathrm{F}] \circ[\mathrm{E}] \text {. }
$$

Putting all of this information together, we have now constructed the $\mathfrak{s l}_{2}$-representation $V_{1} \otimes V_{1}$ on the Grothendieck groups of these categories.

In fact, we have done something stronger. Without too much effort, one could see that these equalities of operators really come from isomorphisms of functors. For example, on $\mathcal{V}_{0}$, there are isomorphisms

$$
\mathrm{F} \circ \mathrm{E} \cong 1 \oplus 1, \quad \mathrm{E} \circ \mathrm{F} \cong 1 \oplus 1,
$$

which imply $\mathrm{E} \circ \mathrm{F} \cong \mathrm{F} \circ \mathrm{E}$. This is referred to as a weak categorification of the $\mathfrak{S l}_{2}$-representation $V_{1}^{\otimes 2}$. For a stronger notion we study the transformations between these functors.

The functors $\mathrm{E}$ and $\mathrm{F}$ are given by tensoring with bimodules $A(2)$ or (2) $A$. These bimodules have endomorphisms $x$ given by multiplication by the path $(2|1| 2)$.

The functor $\mathrm{E} \circ \mathrm{E}$ is given by tensoring with the $(\mathbb{C}, \mathbb{C})$ bimodule (2) $A(2)$. This functor has an endomorphism $\psi$ defined by mapping $(2|1| 2) \mapsto(2)$ and sending all other basis elements to zero. On the functor $\mathrm{E} \circ \mathrm{E}$, consider the natural transformations $x_{1}=x \otimes 1$ and $x_{2}=-1 \otimes x$ coming from the bimodule endomorphism $x$. Then one could check that

$$
x_{1} \psi-\psi x_{2}=\operatorname{Id}_{\mathrm{EoE}}=\psi x_{1}-x_{2} \psi .
$$

There is a diagrammatic calculus which describes these natural transformations where $\psi$ is represented as a crossing and $x$ by a dotted strand. Some of the relations that these natural transformations satisfy can then be expressed as follows:

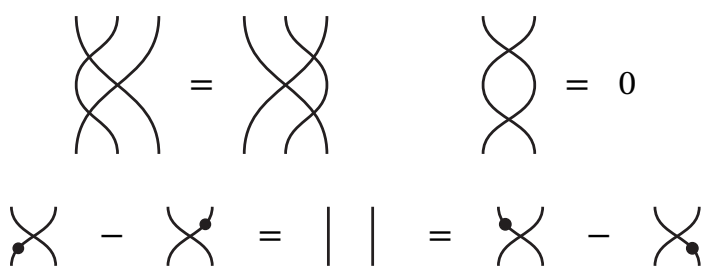

The last line encodes (4.2). This diagrammatic calculus is a graphical description of an example of a nilHecke algebra. In general, nilHecke algebras describe natural transformations in categorifications of $V_{1}^{\otimes n}$.

The importance of controlling the isomorphisms of functors goes back to the original ideas of categorification, but the first explicit form of some of these isomorphisms were discovered by Chuang and Rouquier [CR08] who applied these results to prove the Broué abelian defect conjecture for symmetric groups. They emphasized the role of the affine Hecke algebra (an algebra closely related to the nilHecke algebra) in $\mathfrak{g l}_{2}$-categorification. The first signs that this algebra plays a categorical significance goes back to work of Ariki and Grojnowski who showed that one could recover halves of universal enveloping algebras by considering Grothendieck groups of affine Hecke algebras over all ranks, extending earlier work of Lascoux, Leclerc, and Thibon.

Building upon this, Lauda constructed a 2-category whose Grothendieck group is quantum $\mathfrak{S l}_{2}$. Higher rank versions of this construction were then given by Khovanov and Lauda [KL10] and Rouquier [Rou12] based on a new class of algebras. A remarkable isomorphism between algebras introduced by Khovanov, Lauda, and Rouquier and versions of the affine Hecke algebra was then constructed by Brundan and Kleshchev, and independently Rouquier [BK09, Rou12].

Beyond its role in proving integrality and positivity properties for ordinary quantum groups, one of the main advantages of categorified representation theory has been its role in defining categorical braid group actions. The equivalences introduced by Chuang and Rouquier can be interpreted as the action of elementary generators for a braid group action. Cautis, Kamnitzer, and Licata proved that these equivalences satisfy braid relations in an appropriate category of complexes, showing that any time the categorified quantum group acts on a category, one obtains a categorical action of the braid group. These braid group actions control the categorifications of knot invariants introduced in Section 3.3 and give a representationtheoretic explanation for the existence of Khovanov's categorification of the Jones polynomial discussed in the next section.

Just as quantum groups give rise to especially nice categories of representations, it is expected that categorified quantum groups will have analogously nice 2-categories of representations with the multiplicative structure needed for Crane and Frenkel's approach to 4-dimensional TQFTs. Currently, there is still much work to be done to understand a categorical operation that inputs two categorified representations and outputs a tensor product of categorified representations. Rouquier has developed machinery to define tensor product categorifications, and recent work of Manion and Rouquier connect these ideas to HeegardFloer theory.

4.2. Khovanov homology. The success in categorifying representations used in the Reshetikhin-Turaev construction from Section 3.3 was strong indication that these link invariants themselves might admit categorification. Khovanov gave the first such construction by lifting the Jones polynomial to a homology theory. The Khovanov homology of a link $L$ is the homology of a chain complex of graded vector spaces $K h^{i}(L)$. The complex $K h^{\bullet}(L)$ has graded Euler characteristic equal to the Jones polynomial.

The definition of Khovanov homology of a link follows very closely to the definition of the Kauffman bracket of 
the link. Each crossing of a link has two possible resolutions. Thus, for a link with $n$ crossings, there are $2^{n}$ states of collections of circles. Instead of assigning a factor of $q+q^{-1}$ to each circle in a fixed state, Khovanov assigned the 2dimensional Frobenius algebra $C=\mathbb{Z}[x] /\left(x^{2}\right)$, where the degrees of 1 and $x$ are -1 and 1 , respectively. Multiplication of the factors $q+q^{-1}$ in a particular state is replaced by a tensor product of the algebras $C$. Rather than taking a signed sum of these quantities over all the states, Khovanov organized the tensor products of $C$ into a complex with differentials coming from multiplication and a dual comultiplication on $C$.

We next show how to calculate the Khovanov homology of a Hopf link (ignoring some overall grading shifts).

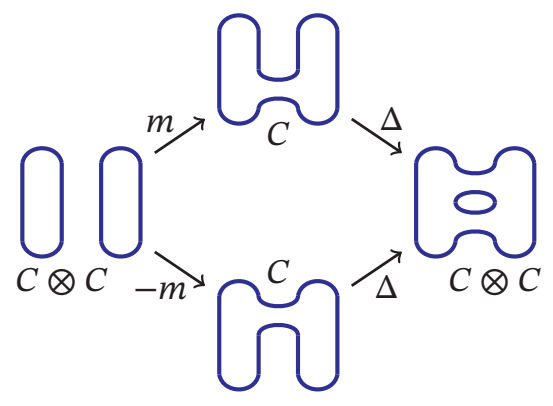

In the leftmost homological degree, we calculate that the kernel is spanned by $\{x \otimes 1-1 \otimes x, x \otimes x\}$. There is no homology in the middle degree and the homology in the rightmost degree is spanned by $\{1 \otimes 1, x \otimes 1-1 \otimes x\}$. Therefore the graded Euler characteristic is equal to $q^{2}\left(1+q^{2}\right)-$ $0+\left(1+q^{-2}\right)$, where the factor of $q^{2}$ in the front of the first term comes from a grading shift built into the theory. This matches up with the calculation of the Kauffman bracket in Figure 3.

- This invariant is strictly stronger than the Jones polynomial. (It can distinguish more knots.)

- This invariant is functorial; i.e., it extends to cobordisms between knots.

- Utilizing functoriality and a deformation of Khovanov homology discovered by Lee, Rasmussen constructed what is known as the s-invariant [Ras10] which led to a new proof of the Milnor conjecture and many other recent novel results in low-dimensional topology. This includes work of Lambert-Cole, Manolescu, Marengon, Piccirillo, Sarkar, and Willis who have proven genuinely new topological results, showing that Khovanov homology can lead to topological applications beyond those coming from gauge-theoretic techniques.

Bar-Natan gave a more topological description of Khovanov homology [BN05]. The role of cobordisms between circles is emphasized and this is really in the spirit of
Crane and Frenkel's categorification philosophy. Stroppel constructed a knot homology coming from category $\mathcal{O}$ [Str05], and later proved it is equivalent to Khovanov homology. Another early categorification of the Jones polynomial was Cautis and Kamnitzer's knot homology using categories of sheaves on the affine Grassmannian [CK08]. Later, Webster used higher representation theory to categorify all Reshetikhen-Turaev invariants [Web17]. In particular, Khovanov homology can be understood from the categorified representation theory of $\mathfrak{s l}_{2}$, analogous to the Reshetikhin-Turaev construction.

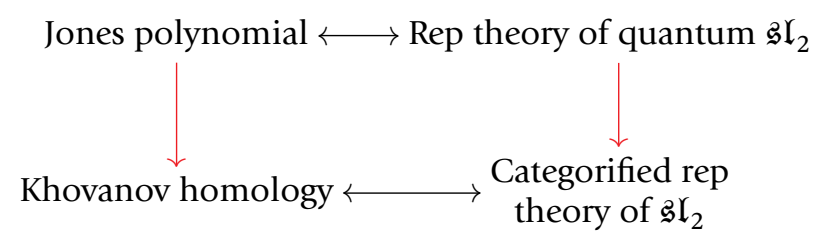

\section{Perspectives}

While the categorification program has led to important applications in group theory, representation theory, and topology, the most ambitious goals laid out by Crane and Frenkel still remain open. The invariants of 3-manifolds and their resulting TQFTs described earlier use representation theory of quantum groups at roots of unity. While categorification of quantum groups and their representations at a generic value of the quantum parameter have been nearly fully developed, the root of unity case remains largely open.

Khovanov proposed studying homological algebra where the role of the differential is replaced by a finitedimensional Hopf algebra as a means of categorification at prime roots of unity [Kho16]. Over a field $\mathbb{k}$ of characteristic $p$, the stable category of modules of the Hopf algebra $\mathbb{k}[\partial] /\left(\partial^{p}\right)$ categorifies the cyclotomic ring $\mathcal{O}_{p}$. Quantum groups where the quantum parameter is specialized to a prime $p$ th root of unity are algebras over $\mathcal{O}_{p}$, and using this framework Elias, Khovanov, and Qi have categorified quantum $\mathfrak{s l}_{2}$ at prime roots of unity. Building upon this, Khovanov, Qi, and Sussan set up machinery to categorify tensor products of representations of this quantum group. In another direction, Qi and Sussan categorified the Jones polynomial evaluated at a prime root of unity [QS20]. These results bring the prospect of categorifying quantum 3-manifold invariants within reach.

There are still many open interesting questions about $\mathfrak{s l}_{2}$ knot homology when the quantum parameter is generic. Cooper and Krushkal [CK12] and Rozansky [Roz14] categorified the colored Jones polynomial by categorifying a Jones-Wenzl projector in the set up of Khovanov homology. This homology turns out to be infinite-dimensional and there are many interesting conjectures about these homologies and connections to 
representation theory of infinite-dimensional Lie algebras [GOR13]. Hogancamp made some progress towards calculations of these homologies [Hog19] that has led to interesting ideas about categorical idempotents due to Elias and Hogancamp.

We have omitted many other important perspectives on categorification such as Khovanov and Rozansky's categorification of the HOMFLYPT polynomial. This construction extends Soergel's pioneering work on algebraic categorifications of Hecke algebras using categories of bimodules which are now known as Soergel bimodules. Rouquier used Soergel bimodules to construct categorical braid group actions that were then used by Khovanov and Rozansky in their construction of HOMFLYPT homology. Soergel bimodules have played a large role in representation theory in the last several years including Elias and Williamson's new proof of the Kazhdan-Lusztig conjecture [EW14]. This field has many exciting directions including connections with Hilbert schemes, mathematical physics, and the combinatorics of symmetric functions.

It is clear that the fundamental ideas of categorification envisioned by Crane and Frenkel have now become integrated into many areas of modern mathematics and theoretical physics and have been leveraged in many directions to make new advances. We hope that this invitation to categorification has encouraged the reader to similarly seek higher structure in their mathematical pursuits.

ACKNOWLEDGMENTS. The authors would like to thank John Baez, Igor Frenkel, and Mikhail Khovanov for introducing them to the ideas presented in this paper. They are also grateful to Raphaël Rouquier, Catharina Stroppel, and Ben Webster for helpful comments. The first author was partially supported by NSF grant DMS-1902092 and Army Research Office W911NF-201-0075. The second author was partially supported by NSF grant DMS-1807161 and PSC CUNY Award 640120052 .

\section{References}

[BFK99] Joseph Bernstein, Igor Frenkel, and Mikhail Khovanov, A categorification of the Temperley-Lieb algebra and Schur quotients of $U\left(\mathfrak{g}_{2}\right)$ via projective and Zuckerman functors, Selecta Math. (N.S.) 5 (1999), no. 2, 199-241, DOI 10.1007/s000290050047. MR1714141

[BK09] Jonathan Brundan and Alexander Kleshchev, Blocks of cyclotomic Hecke algebras and Khovanov-Lauda algebras, Invent. Math. 178 (2009), no. 3, 451-484, DOI 10.1007/s00222-009-0204-8. MR2551762

[BL11] John C. Baez and Aaron D. Lauda, A prehistory of n-categorical physics, Deep Beauty, Cambridge University Press, Cambridge, 2011, pp. 13-128. MR2752518
[BN05] Dror Bar-Natan, Khovanov's homology for tangles and cobordisms, Geom. Topol. 9 (2005), 1443-1499, DOI 10.2140/gt.2005.9.1443. MR2174270

[CF94] Louis Crane and Igor B. Frenkel, Four-dimensional topological quantum field theory, Hopf categories, and the canonical bases, J. Math. Phys. 35 (1994), no. 10, 5136-5154, DOI 10.1063/1.530746. Topology and physics. MR1295461

[CK08] Sabin Cautis and Joel Kamnitzer, Knot homology via derived categories of coherent sheaves. I. The $\mathfrak{s l}(2)$ case, Duke Math. J. 142 (2008), no. 3, 511-588, DOI 10.1215/00127094-2008-012. MR2411561

[CK12] Benjamin Cooper and Vyacheslav Krushkal, Categorification of the Jones-Wenzl projectors, Quantum Topol. 3 (2012), no. 2, 139-180, DOI 10.4171/QT/27. MR2901969

[CR08] Joseph Chuang and Raphaël Rouquier, Derived equivalences for symmetric groups and $\mathfrak{S l}_{2}$-categorification, Ann. of Math. (2) 167 (2008), no. 1, 245-298, DOI 10.4007/annals.2008.167.245 MR2373155

[EW14] Ben Elias and Geordie Williamson, The Hodge theory of Soergel bimodules, Ann. of Math. (2) 180 (2014), no. 3, 1089-1136, DOI 10.4007/annals.2014.180.3.6. MR3245013

[FKS06] Igor Frenkel, Mikhail Khovanov, and Catharina Stroppel, A categorification of finite-dimensional irreducible representations of quantum $\mathfrak{S l}_{2}$ and their tensor products, Selecta Math. (N.S.) 12 (2006), no. 3-4, 379-431, DOI 10.1007/s00029-007-0031-y. MR2305608

[GOR13] Eugene Gorsky, Alexei Oblomkov, and Jacob Rasmussen, On stable Khovanov homology of torus knots, Exp. Math. 22 (2013), no. 3, 265-281, DOI 10.1080/10586458.2013.798553. MR3171092

[Hog19] Matthew Hogancamp, A polynomial action on colored $\mathfrak{S I}_{2}$ link homology, Quantum Topol. 10 (2019), no. 1, 1-75, DOI 10.4171/QT/122 MR3900776

[Kho16] Mikhail Khovanov, Hopfological algebra and categorification at a root of unity: the first steps, J. Knot Theory Ramifications 25 (2016), no. 3, 1640006, 26, DOI 10.1142/S021821651640006X. MR3475073

[KL10] Mikhail Khovanov and Aaron D. Lauda, A categorification of quantum $\mathrm{sl}(n)$, Quantum Topol. 1 (2010), no. 1, 1-92, DOI $10.4171 / \mathrm{QT} / 1$. MR2628852

[QS20] You Qi and Joshua Sussan, On some p-differential graded link homologies, 2020.

[Ras10] Jacob Rasmussen, Khovanov homology and the slice genus, Invent. Math. 182 (2010), no. 2, 419-447, DOI 10.1007/s00222-010-0275-6 MR2729272

[Rou12] Raphaël Rouquier, Quiver Hecke algebras and 2-Lie algebras, Algebra Colloq. 19 (2012), no. 2, 359-410, DOI 10.1142/S1005386712000247. MR2908731

[Roz14] Lev Rozansky, An infinite torus braid yields a categorified Jones-Wenzl projector, Fund. Math. 225 (2014), no. 1, 305-326, DOI 10.4064/fm225-1-14, MR3205575

[Str05] Catharina Stroppel, Categorification of the TemperleyLieb category, tangles, and cobordisms via projective functors, Duke Math. J. 126 (2005), no. 3, 547-596, DOI 10.1215/S0012-7094-04-12634-X. MR2120117

[Web17] Ben Webster, Knot invariants and higher representation theory, Mem. Amer. Math. Soc. 250 (2017), no. 1191, v+141, DOI $10.1090 / \mathrm{memo} / 1191$. MR3709726 


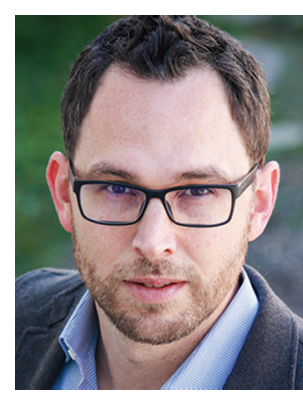

Aaron D. Lauda

\section{Credits}

Opening image is courtesy of bgblue via Getty. All article figures are courtesy of the authors.

Photo of Aaron D. Lauda is courtesy of Alan Mamaril.

Photo of Joshua Sussan is courtesy of the Graduate Center/CUNY.

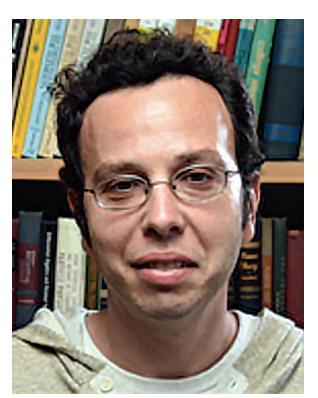

Joshua Sussan

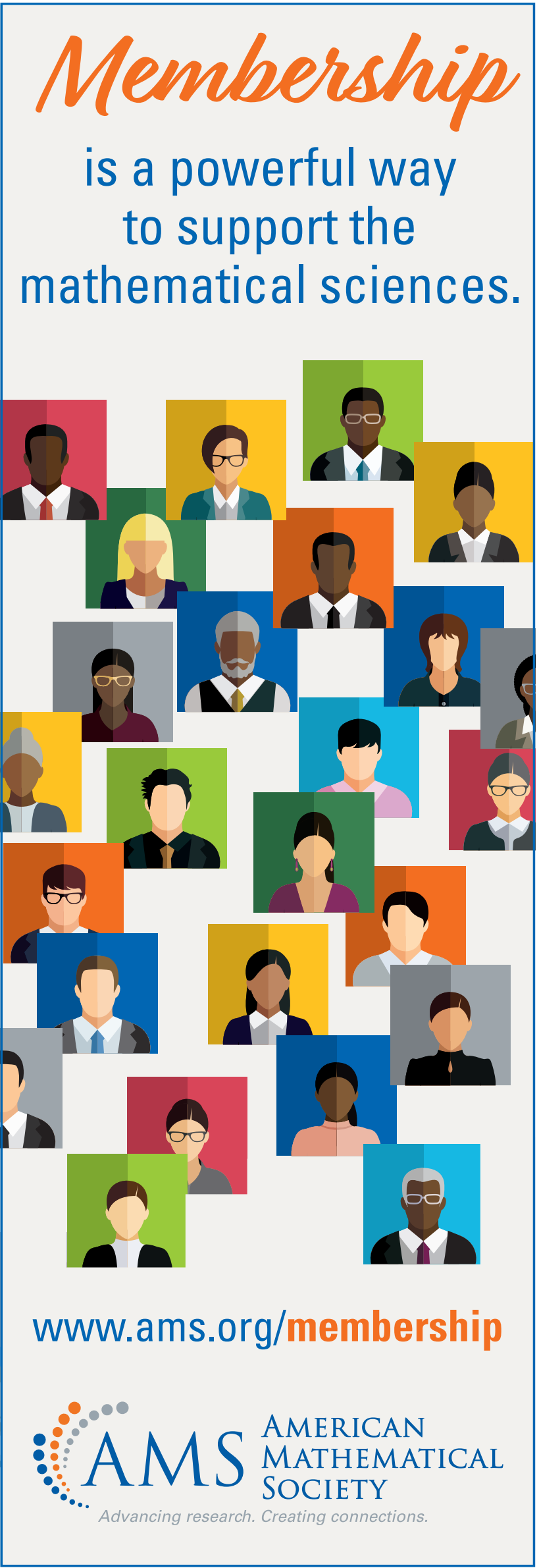

